\title{
When Can You Tile a Box With Translates of Two Given Rectangular Bricks?
}

\author{
Richard J. Bower and T. S. Michael* \\ Mathematics Department, United States Naval Academy \\ Annapolis, MD 21402 \\ tsm@usna.edu
}

Submitted: Feb 21, 2004; Accepted: May 10, 2004; Published: May 14, 2004

MR Subject Classifications: 05B45, 52C22

\begin{abstract}
When can a $d$-dimensional rectangular box $R$ be tiled by translates of two given $d$-dimensional rectangular bricks $B_{1}$ and $B_{2}$ ? We prove that $R$ can be tiled by translates of $B_{1}$ and $B_{2}$ if and only if $R$ can be partitioned by a hyperplane into two sub-boxes $R_{1}$ and $R_{2}$ such that $R_{i}$ can be tiled by translates of the brick $B_{i}$ alone $(i=1,2)$. Thus an obvious sufficient condition for a tiling is also a necessary condition. (However, there may be tilings that do not give rise to a bipartition of R.)

There is an equivalent formulation in terms of the (not necessarily integer) edge lengths of $R, B_{1}$, and $B_{2}$. Let $R$ be of size $z_{1} \times z_{2} \times \cdots \times z_{d}$, and let $B_{1}$ and $B_{2}$ be of respective sizes $v_{1} \times v_{2} \times \cdots \times v_{d}$ and $w_{1} \times w_{2} \times \cdots \times w_{d}$. Then there is a tiling of the box $R$ with translates of the bricks $B_{1}$ and $B_{2}$ if and only if

(a) $z_{i} / v_{i}$ is an integer for $i=1,2, \ldots, d$; or

(b) $z_{i} / w_{i}$ is an integer for $i=1,2, \ldots, d$; or

(c) there is an index $k$ such that $z_{i} / v_{i}$ and $z_{i} / w_{i}$ are integers for all $i \neq k$, and $z_{k}=\alpha v_{k}+\beta w_{k}$ for some nonnegative integers $\alpha$ and $\beta$.

Our theorem extends some well known results (due to de Bruijn and Klarner) on tilings of rectangles by rectangles with integer edge lengths.
\end{abstract}

\section{Introduction and Main Theorem}

A $d$-dimensional rectangular box or brick of size $v_{1} \times v_{2} \times \cdots \times v_{d}$ is any translate of the set

$$
\left\{\left(x_{1}, x_{2}, \ldots, x_{d}\right) \in \mathbf{R}^{d}: 0 \leq x_{i} \leq v_{i} \text { for } i=1,2, \ldots, d\right\} .
$$

\footnotetext{
*Corresponding author. Partially supported by the Naval Academy Research Council
} 
Thus a box or brick in dimension $d=2$ is simply a rectangle with sides parallel to the coordinate axes. We study the problem of tiling a $d$-dimensional rectangular box with translates of two given $d$-dimensional rectangular bricks. We use the term tile in the following sense: The interiors of the bricks must be disjoint, and their union must be the entire box.

We will provide two different characterizations of the boxes that can be tiled by translates of two given bricks. One characterization is geometric. The other is arithmetic and involves the edge lengths of the bricks and the box. We do not require that the bricks and the box have integer edge lengths, although this special case is a crucial element of our analysis. Our main theorem extends several 2-dimensional tiling theorems in a pleasing manner.

Tilings of a box with translates of a single brick are readily characterized. We say that the $z_{1} \times z_{2} \times \cdots \times z_{d}$ box is a multiple of the $v_{1} \times v_{2} \times \cdots \times v_{d}$ brick provided $z_{i} / v_{i}$ is an integer for $i=1,2, \ldots, d$. The following observation is clear.

Observation. The $d$-dimensional box $R$ can be tiled by translates of a given brick $B$ if and only if $R$ is a multiple of $B$. Moreover, any such tiling is unique.

When we have two bricks at our disposal, the situation is more complicated. Note that a tiling of a box with translates of two bricks need not be unique. For instance, Figure 1 shows two tilings of a box $R$ with translates of two rectangular bricks $B_{1}$ and $B_{2}$. In (a) the box $R$ is partitioned by a plane into two sub-boxes $R_{1}$ and $R_{2}$, and the sub-box $R_{i}$ is a multiple of the brick $B_{i}$ for $i=1,2$. We refer to such a tiling as a bipartite tiling of $R$ with $B_{1}$ and $B_{2}$. In (b) we exhibit a non-bipartite tiling of $R$ with the same bricks $B_{1}$ and $B_{2}$. Because the trivial box of size $0 \times 0 \times \cdots \times 0$ is a multiple of every non-trivial $d$-dimensional box, either of the two sub-boxes may be trivial in a bipartite tiling of a box $R$; this degenerate situation occurs precisely when $R$ is a multiple of $B_{1}$ or $B_{2}$.

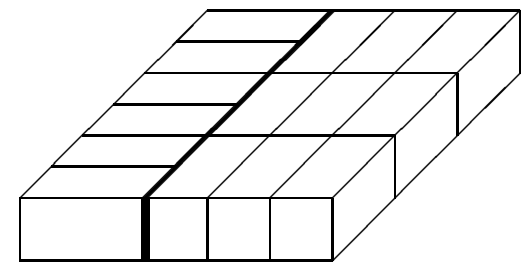

(a)

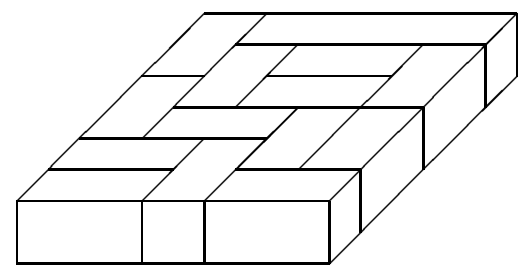

(b)

Figure 1: (a) A bipartite tiling (b) A non-bipartite tiling

Clearly, the existence of a bipartite tiling is sufficient for the existence of a tiling of a box with translates of two given bricks. The thrust of our main theorem is that this obvious sufficient condition is also necessary:

Theorem 1 (Geometric). The $d$-dimensional box $R$ can be tiled by translates of two given $d$-dimensional bricks $B_{1}$ and $B_{2}$ if and only if $R$ can be partitioned by a hyperplane into two sub-boxes $R_{1}$ and $R_{2}$ such that $R_{i}$ is a multiple of $B_{i}$ for $i=1,2$. 
We emphasize that Theorem 1 does not say that every tiling must be bipartite. However, the existence of a non-bipartite tiling implies the existence of bipartite tiling.

Theorem 1 gives a satisfying and complete geometric characterization of the boxes that can be tiled by translates of two given bricks. We now provide an equivalent arithmetic characterization in terms of the edge lengths of the box and the bricks. For real numbers $v$ and $w$ we denote the set of all nonnegative integer linear combinations of $v$ and $w$ by

$$
\langle v, w\rangle=\{\alpha v+\beta w: \alpha=0,1,2, \ldots, \beta=0,1,2, \ldots\} .
$$

Theorem $\mathbf{1}^{\prime}$ (Arithmetic). Let $z_{i}, v_{i}$, and $w_{i}$ be positive real numbers for $i=1,2, \ldots, d$. There is a tiling of a $z_{1} \times z_{2} \times \cdots \times z_{d}$ box with translates of $v_{1} \times v_{2} \times \cdots \times v_{d}$ and $w_{1} \times w_{2} \times \cdots \times w_{d}$ bricks if and only if

(a) $z_{i} / v_{i}$ is an integer for $i=1,2, \ldots, d$; or

(b) $z_{i} / w_{i}$ is an integer for $i=1,2, \ldots, d$; or

(c) there is an index $k$ such that $z_{k}$ is in $\left\langle v_{k}, w_{k}\right\rangle$, and the numbers $z_{i} / v_{i}$ and $z_{i} / w_{i}$ are integers for all $i \neq k$.

Condition (a) or (b) holds when the box is tiled by translates of one of the bricks, while (c) holds when a tiling uses both bricks.

Example. It is possible to tile a $12 \times 12 \times 11$ box with $4 \times 4 \times 4$ and $3 \times 3 \times 3$ cubical bricks. Condition (c) of Theorem $1^{\prime}$ is satisfied (with $k=3$ ) because $12 / 4$ and $12 / 3$ are both integers, and $11=2 \cdot 4+1 \cdot 3$. Figure 2 shows a bipartite tiling. The two sub-boxes are of sizes $12 \times 12 \times 8$ and $12 \times 12 \times 3$.

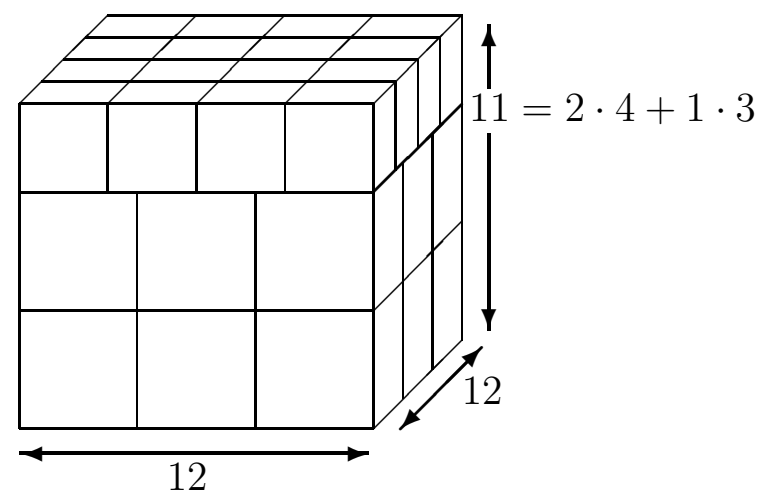

Figure 2: A tiling of a $12 \times 12 \times 11$ box with $4 \times 4 \times 4$ and $3 \times 3 \times 3$ cubical bricks

The preceding example helps reveal the equivalence of Theorem 1 and Theorem $1^{\prime}$. The two sub-boxes $R_{1}$ and $R_{2}$ in Theorem 1 are non-trivial and are separated by a hyperplane perpendicular to the $k$-th coordinate axis exactly when $k$ is an index for which condition (c) holds in Theorem $1^{\prime}$. The integrality conditions imposed on $z_{i} / v_{i}$ and $z_{i} / w_{i}$ for $i \neq k$ guarantee that that $R_{1}$ and $R_{2}$ are multiples of the two respective bricks.

The arithmetic conditions in Theorem $1^{\prime}$ supply us with an algorithm to recognize when a $z_{1} \times z_{2} \times \cdots \times z_{d}$ box can be tiled by translates of $v_{1} \times v_{2} \times \cdots \times v_{d}$ and $w_{1} \times w_{2} \times \cdots \times w_{d}$ 
bricks. Integrality of the $2 d$ real numbers $z_{i} / v_{i}$ and $z_{i} / w_{i}$ for $i=1,2, \ldots, d$ is readily checked, and $z_{k}$ is in $\left\langle v_{k}, w_{k}\right\rangle$ if and only if the real number $\left(z_{k}-\alpha v_{k}\right) / w_{k}$ is an integer for some $\alpha$ in $\left\{0,1, \ldots,\left\lfloor z_{k} / v_{k}\right\rfloor\right\}$.

Sections 2 through 6 contain preliminary results and a discussion of important special cases. The proof of Theorem $1^{\prime}$ is in Section 7 . Our discussion is elementary and accessible to a wide audience.

\section{Re-Scaling and a Counting Lemma}

We begin with two basic lemmas on tilings. The first result is easy, and we omit the proof.

Lemma 2. Let $h$ be a positive real number. Then there is a tiling of a $z_{1} \times z_{2}$ rectangle with translates of $v_{1} \times v_{2}$ and $w_{1} \times w_{2}$ rectangular bricks if and only if there is a tiling of an $\left(h z_{1}\right) \times z_{2}$ rectangle with translates of $\left(h v_{1}\right) \times v_{2}$ and $\left(h w_{1}\right) \times w_{2}$ rectangular bricks.

The number $h$ represents a re-scaling factor applied to all horizontal edge lengths of the rectangles. There is a corresponding result for vertical re-scalings, as well as an extension to re-scalings in higher dimensions.

Our second basic result uses a counting argument to obtain a fundamental necessary condition for a box to be tiled by translates of two given bricks.

Lemma 3. Suppose that there is a tiling of a $z_{1} \times z_{2} \times \cdots \times z_{d}$ box with translates of $v_{1} \times v_{2} \times \cdots \times v_{d}$ and $w_{1} \times w_{2} \times \cdots \times w_{d}$ bricks in $d$ dimensions. Then there are nonnegative integers $\alpha_{i}$ and $\beta_{i}$ such that $z_{i}=\alpha_{i} v_{i}+\beta_{i} w_{i}$ for $i=1,2, \ldots, d$. Moreover, if $v_{i}$ is irrational and $w_{i}$ is rational, then $\alpha_{i}$ and $\beta_{i}$ are unique.

Proof. Count the number of bricks of each type incident with an edge of length $z_{i}$ (parallel to the $i$-th coordinate axis) of the box. If there are $\alpha_{i}$ bricks of size $v_{1} \times v_{2} \times \cdots \times v_{d}$ and $\beta_{i}$ bricks of size $w_{1} \times w_{2} \times \cdots \times w_{d}$, then this count shows that $z_{i}=\alpha_{i} v_{i}+\beta_{i} w_{i}$.

Suppose that $v_{i}$ is irrational and $w_{i}$ is rational. Let $\alpha_{i}, \beta_{i}, \alpha_{i}^{\prime}$, and $\beta_{i}^{\prime}$ be nonnegative integers such that $z_{i}=\alpha_{i} v_{i}+\beta_{i} w_{i}=\alpha_{i}^{\prime} v_{i}+\beta_{i}^{\prime} w_{i}$. Then $\left(\alpha_{i}-\alpha_{i}^{\prime}\right) v_{i}=\left(\beta_{i}^{\prime}-\beta_{i}\right) w_{i}$. The expression on the right is rational, and thus $\alpha_{i}^{\prime}=\alpha_{i}$. Then $\beta_{i}^{\prime}=\beta_{i}$, and the uniqueness of $\alpha_{i}$ and $\beta_{i}$ is established.

The following elementary result tells us that the necessary counting condition of Lemma 3 is also sufficient for the tiling of an interval by translates of two given intervals on the real line. Thus our main theorem is true in dimension 1.

Theorem 4. Let $z, v$, and $w$ be positive real numbers. The following statements are equivalent.

(a) An interval of length $z$ can be tiled by translates of intervals of length $v$ and $w$.

(b) An interval of length $z$ has a bipartite tiling with intervals of length $v$ and $w$.

(c) There are nonnegative integers $\alpha$ and $\beta$ such that $z=\alpha v+\beta w$.

Proof. If an interval of length $z$ is tiled by $\alpha$ intervals of length $v$ and $\beta$ intervals of length $w$, then $z=\alpha v+\beta w$, and we may place $\alpha$ intervals of length $v$ followed by $\beta$ intervals of length $w$ to produce a bipartite tiling. Thus (a) implies (b) and (c). This construction also makes it clear that (c) implies (a). 


\section{The Divisibility Lemma for Tilings}

In an integer rectangle the length of each edge is an integer. The next result is a key step in our proof of Theorem $1^{\prime}$.

Divisibility Lemma. Let $v$ and $w$ be positive integers. Suppose that the integer rectangle $R$ is tiled by integer rectangular bricks, each of which has width divisible by $v$ or height divisible by $w$. Then $R$ itself has width divisible by $v$ or height divisible by $w$.

Generalizations and variations of the divisibility lemma appear throughout the literature on tiling. For example, the divisibility lemma can be deduced from Wagon's [9] result on "semi-integer rectangles" by using a re-scaling argument. To keep our discussion self-contained we include a charge-counting proof of the divisibility lemma. The related checkerboard coloring scheme $[4,5,8,9]$ and polynomial factorizations $[1,3,6]$ also work.

Proof. Let $R$ be an $n_{1} \times n_{2}$ rectangle with a tiling of the specified type. Partition $R$ into $n_{1} n_{2}$ unit cells with segments parallel to the edges. Index the rows $1,2, \ldots, n_{2}$ from bottom to top. Place a unit positive charge in each of the $n_{1}$ cells in row $j$ for $j=1, w+1,2 w+1, \ldots$ and a unit negative charge in each of the $n_{1}$ cells in row $j$ for $j=w, 2 w, 3 w, \ldots$, as in Figure 3. All other cells in $R$ receive charge 0 .

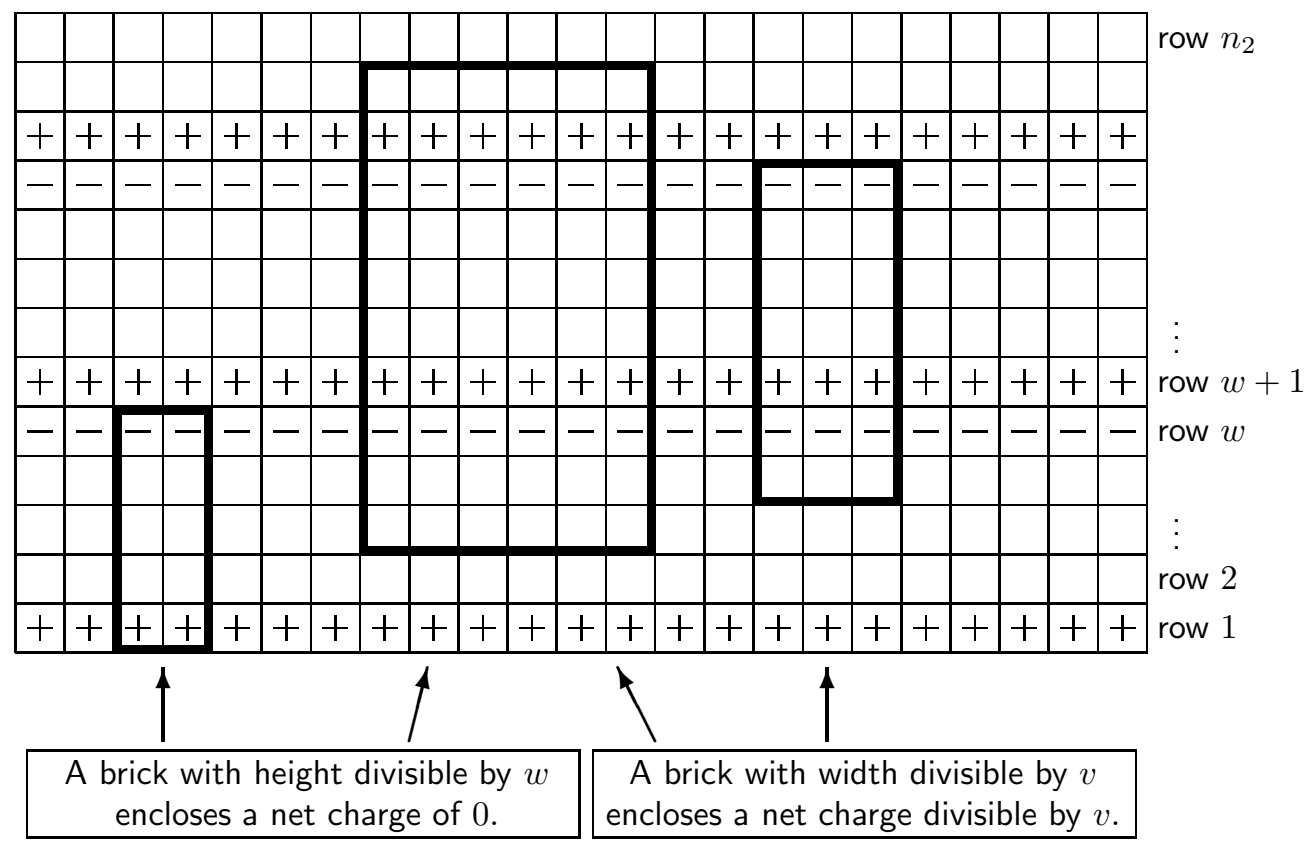

Figure 3: A charge-counting argument, illustrated for $v=3$ and $w=5$

Consider the net charge enclosed by $R$ and by each rectangular brick. Observe that the net charge of the entire rectangle $R$ equals $n_{1}$ if $w$ does not divide $n_{2}$ and equals 0 if $w$ does divide $n_{2}$. Now each rectangular brick with height divisible by $w$ encloses a net 
charge of 0 , while each rectangular brick with width divisible by $v$ encloses a net charge that is divisible by $v$. It follows that if $w$ does not divide $n_{2}$, then $v$ must divide $n_{1}$.

\section{Tiling with Integer Rectangles}

We now state and prove Theorem $1^{\prime}$ for integer rectangles.

Theorem 5. Let $v_{1}, v_{2}, w_{1}$, and $w_{2}$ be positive integers with $\operatorname{gcd}\left(v_{1}, w_{1}\right)=\operatorname{gcd}\left(v_{2}, w_{2}\right)=$ 1. Then an integer rectangle of size $n_{1} \times n_{2}$ can be tiled by translates of $v_{1} \times v_{2}$ and $w_{1} \times w_{2}$ rectangular bricks if and only if

(a) $v_{1}$ divides $n_{1}$, and $v_{2}$ divides $n_{2} ;$ or

(b) $w_{1}$ divides $n_{1}$, and $w_{2}$ divides $n_{2} ;$ or

(c) $v_{1} w_{1}$ divides $n_{1}$, and $n_{2}$ is in $\left\langle v_{2}, w_{2}\right\rangle$; or

(d) $v_{2} w_{2}$ divides $n_{2}$, and $n_{1}$ is in $\left\langle v_{1}, w_{1}\right\rangle$.

Proof. Let $B_{1}$ and $B_{2}$ be $v_{1} \times v_{2}$ and $w_{1} \times w_{2}$ rectangular bricks, respectively, and let $R$ be an $n_{1} \times n_{2}$ rectangle. Suppose that $R$ is tiled by translates of $B_{1}$ and $B_{2}$. Lemma 3 tells us that $n_{1}$ is in $\left\langle v_{1}, w_{1}\right\rangle$ and $n_{2}$ is in $\left\langle v_{2}, w_{2}\right\rangle$. The brick $B_{1}$ has width $v_{1}$, while $B_{2}$ has height $w_{2}$. The divisibility lemma implies that $v_{1}$ divides $n_{1}$, or $w_{2}$ divides $n_{2}$. Similarly, $B_{2}$ has width $w_{1}$, while $B_{1}$ has height $v_{2}$, and so the divisibility lemma implies that $w_{1}$ divides $n_{1}$, or $v_{2}$ divides $n_{2}$. It follows that one of the four conditions (a)-(d) must hold.

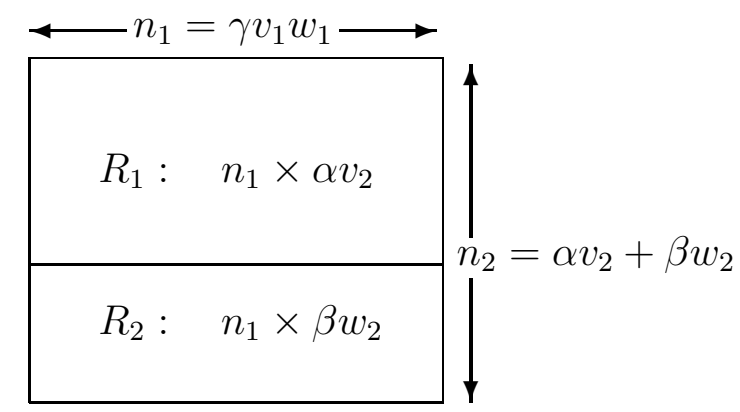

Figure 4: The proof of Theorem 5

We now show that $R$ can be tiled by translates of $B_{1}$ and $B_{2}$ if any of (a)-(d) holds. If (a) or (b) holds, then $R$ is a multiple of $B_{1}$ or $B_{2}$, and the desired tiling certainly exists. Suppose that (c) holds, where $n_{1}=\gamma v_{1} w_{1}$ and $n_{2}=\alpha v_{2}+\beta w_{2}$. Then a horizontal line partitions $R$ into a rectangle $R_{1}$ of size $n_{1} \times \alpha v_{2}$ and a rectangle $R_{2}$ of size $n_{1} \times \beta w_{2}$, as in Figure 4. Now $R_{1}$ is a multiple of the brick $B_{1}$, while $R_{2}$ is a multiple of the brick $B_{2}$. Thus $R$ has a bipartite tiling with $B_{1}$ and $B_{2}$. Condition $(\mathrm{d})$ is treated similarly.

As is clear from the proof of Theorem 5, each of the conditions (a)-(d) implies the existence of a bipartite tiling of $R$ with $B_{1}$ and $B_{2}$. 
The hypothesis that corresponding edge lengths of the bricks be relatively prime is not an obstacle in applying Theorem 5; a re-scaling argument allows us to treat the cases where this hypothesis is not met, as in the proof of Corollary 6 below.

\section{Corollaries}

Theorem 5 contains several important tiling results as special cases.

Corollary 6 (de Bruijn [3] and Klarner [7]). Let $v$ and $w$ be positive integers. An integer rectangle of size $n_{1} \times n_{2}$ can be tiled by $v \times w$ rectangular bricks (with both orientations allowed) if and only if

(a) $v$ divides $n_{1}$ or $n_{2}$; and

(b) $w$ divides $n_{1}$ or $n_{2}$; and

(c) $n_{1}$ is in $\langle v, w\rangle ; \quad$ and

(d) $n_{2}$ is in $\langle v, w\rangle$.

Proof. If $v$ and $w$ are relatively prime, then the result follows from Theorem 5 with $v_{1}=w_{2}=v$ and $w_{1}=v_{2}=w$. If $v$ and $w$ are not relatively prime, then we first divide $n_{1}, n_{2}, v$, and $w$ by $\operatorname{gcd}(v, w)$. The re-scaled rectangle must be an integer rectangle for a tiling to exist, and we are in the previous situation. By Lemma 2 there is a tiling with the original rectangular bricks if and only if there is a tiling with the re-scaled bricks.

We also obtain the following less well known result, which appeared in 1995.

Corollary 7 (Fricke [4]). Let $v$ and $w$ be relatively prime positive integers. An $n_{1} \times n_{2}$ rectangle can be tiled by $v \times v$ and $w \times w$ squares if and only if

(a) $v$ divides $n_{1}$ and $n_{2} ; \quad$ or

(b) $w$ divides $n_{1}$ and $n_{2} ; \quad$ or

(c) $v w$ divides $n_{1}$, and $n_{2}$ is in $\langle v, w\rangle$; or

(d) $v w$ divides $n_{2}$ and $n_{1}$ is in $\langle v, w\rangle$.

Proof. In Theorem 5 let $v_{1}=v_{2}=v$ and $w_{1}=w_{2}=w$.

\section{Tiling Rectangles with Rectangles}

We now extend Theorem 5 to obtain necessary and sufficient conditions for a rectangle to be tiled by translates of two (not necessarily integer) rectangles. In other words, we prove our main theorem in dimension 2 . We will see that the 2-dimensional case is the crucial one for establishing the general theorem.

Theorem 8 (Geometric). The rectangle $R$ can be tiled by translates of two given rectangular bricks $B_{1}$ and $B_{2}$ if and only if $R$ can be partitioned by a line into two sub-rectangles $R_{1}$ and $R_{2}$ such that $R_{i}$ is a multiple of $B_{i}$ for $i=1,2$.

Proof. Clearly, if $R$ can be partitioned into a multiple of $B_{1}$ and a multiple of $B_{2}$, then $R$ has a tiling with translates of $B_{1}$ and $B_{2}$. 
Suppose that $R$ can be tiled by translates of the bricks $B_{1}$ and $B_{2}$. Without loss of generality $B_{1}$ is a $v \times 1$ rectangle and $B_{2}$ is a $1 \times w$ rectangle, as suitable horizontal and vertical re-scalings bring about this situation. Let $R$ be a $z_{1} \times z_{2}$ rectangle and consider a particular tiling of $R$ with translates of $B_{1}$ and $B_{2}$. If this tiling uses translates of only one of the two bricks, then $R$ is a multiple of that brick, and we have our desired (degenerate) bipartition of $R$. We henceforth suppose that the tiling uses translates of both $B_{1}$ and $B_{2}$.

Case 1: Suppose that $v$ and $w$ are both rational. Then after suitable horizontal and vertical re-scalings we may assume that $B_{1}, B_{2}$, and $R$ are integer rectangles and that the corresponding edge lengths of $B_{1}$ and $B_{2}$ are relatively prime. Theorem 5 establishes the existence of the desired bipartite tiling.

Case 2: Suppose that at least one of $v$ and $w$ is irrational. Without loss of generality $v$ is irrational. By Lemma 3

$$
z_{1}=\alpha v+\beta
$$

where $\alpha$ and $\beta$ are unique nonnegative integers. Now a vertical line partitions $R$ into the sub-rectangles $R_{1}$ and $R_{2}$ of respective sizes $(\alpha v) \times z_{2}$ and $\beta \times z_{2}$. We will show that $R_{i}$ is a multiple of the brick $B_{i}$ for $i=1,2$, which will complete the proof.

Claim 1: The sub-rectangle $R_{1}$ of size $(\alpha v) \times z_{2}$ is a multiple of the brick $B_{1}$ of size $v \times 1$. Clearly, $(\alpha v) / v=\alpha$ is an integer. We use a tile-sliding argument to show that $z_{2} / 1=z_{2}$ is an integer, which will establish the claim. First remove the translates of the brick $B_{2}$ from the tiling. Then draw a horizontal line $k$ units from the bottom of $R$ for $k=1,2, \ldots,\left\lfloor z_{2}\right\rfloor$ to slice $R$ into horizontal strips. Beginning from the lowest strip and working upward, we see that (1) implies that each strip in turn wholly contains exactly $\alpha$ copies of the $v \times 1$ brick $B_{1}$. We slide the bricks to the left within each successive strip to tile a portion of the sub-rectangle $R_{1}$ with translates of $B_{1}$, as shown in Figure 5. If $z_{2}$ is not an integer, then in the final step we see that the topmost horizontal strip of size $z_{1} \times\left(z_{2}-\left\lfloor z_{2}\right\rfloor\right)$ must contain $\alpha$ bricks of size $v \times 1$, which is impossible because $0<z_{2}-\left\lfloor z_{2}\right\rfloor<1$. Therefore $z_{2}$ in an integer, and our claim is true.

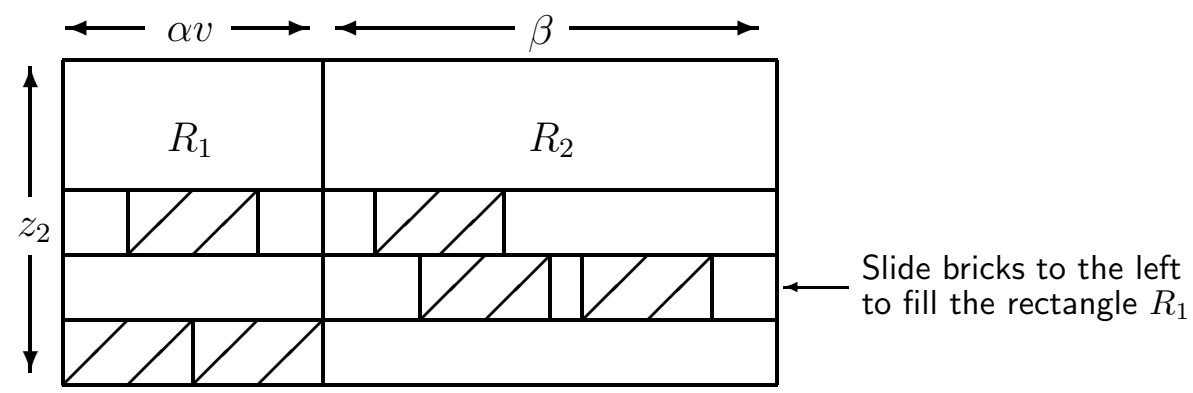

Figure 5: The proof of Claim 1

Claim 2: The sub-rectangle $R_{2}$ of size $\beta \times z_{2}$ is a multiple of the brick $B_{2}$ of size $1 \times w$. The argument is almost identical to the one given above; we remove the bricks $B_{1}$, slice $R$ into strips with horizontal lines at height $w, 2 w, \ldots,\left\lfloor z_{2} / w\right\rfloor w$, and slide the bricks $B_{2}$ to the right to fill $R_{2}$. 
Here is the equivalent arithmetic formulation of Theorem 8; the equivalence is clear from our discussion following the example in Section 1.

Theorem $\mathbf{8}^{\prime}$ (Arithmetic). $A z_{1} \times z_{2}$ rectangle can be tiled by translates of $v_{1} \times v_{2}$ and $w_{1} \times w_{2}$ rectangles if and only if

(a) $z_{1} / v_{1}$ and $z_{2} / v_{2}$ are integers; or

(b) $z_{2} / w_{1}$ and $z_{2} / w_{2}$ are integers; or

(c) $z_{1}$ is in $\left\langle v_{1}, w_{1}\right\rangle$, and the numbers $z_{2} / v_{2}$ and $z_{2} / w_{2}$ are integers; or

(d) $z_{2}$ is in $\left\langle v_{2}, w_{2}\right\rangle$, and the numbers $z_{1} / v_{1}$ and $z_{1} / w_{1}$ are integers.

\section{Proof of Theorem $\mathbf{1}^{\prime}$}

We prove our main theorem in its arithmetic formulation. We have seen that Theorem $1^{\prime}$ is true in dimension 1 (Theorem 4) and dimension 2 (Theorem $8^{\prime}$ ). We henceforth suppose that $d \geq 3$. If either (a) or (b) is true, then the box can be tiled by translates of one brick, while if (c) is true, then there is a bipartite tiling.

Conversely, suppose that a $z_{1} \times z_{2} \times \cdots \times z_{d}$ box $R$ is tiled by translates of bricks of size $v_{1} \times v_{2} \times \cdots \times v_{d}$ and $w_{1} \times w_{2} \times \cdots \times w_{d}$. Also, suppose that neither (a) nor (b) holds. We will show that condition (c) must hold, which will complete the proof. Because (a) and (b) fail, there are indices $j$ and $k$ such that neither $z_{j} / v_{j}$ nor $z_{k} / w_{k}$ is an integer. We claim that $j$ must equal $k$. For if $j \neq k$, then an inspection of a suitable 2-dimensional face of $R$ reveals a tiling of a $z_{j} \times z_{k}$ rectangle with translates of $v_{j} \times v_{k}$ and $w_{j} \times w_{k}$ rectangular bricks. However, each of the conditions in Theorem $8^{\prime}$ fails, and therefore no such tiling exists. Therefore $j=k$. Of course, $z_{k}$ is in $\left\langle v_{k}, w_{k}\right\rangle$ by Lemma 3. We have shown that (c) holds.

\section{References}

[1] P. Boisen, Polynomials and packings: a new proof of de Bruijn's theorem, Discrete Math. 146 (1995), $285-287$.

[2] R.A. Brualdi and T.H. Foregger, tiling boxes with harmonic bricks, J. Combin. Theory B 17 (1974), $81-114$.

[3] N.G. de Bruijn, Filling boxes with bricks, Amer. Math. Monthly 76 (1969), 37-40.

[4] J. Fricke, Quadratzerlegung eines Rechtecks, Math. Semesterber. 42 (1995), 53-62.

[5] S.W. Golomb, Polyominoes. 2nd ed., Princeton Univ. Press, 1994.

[6] J.B. Kelly Polynomials and polyominoes, Amer. Math. Monthly 73 (1966), 464-471.

[7] D.A. Klarner, Tiling a rectangle with congruent $N$-ominoes, J. Combin. Theory A 7 (1969), 107-115.

[8] G.E. Martin, Polyominoes, A Guide to Puzzles and Problems in Tiling. Math. Assoc. of America, 1991.

[9] S. Wagon, Fourteen proofs of a result about tiling a rectangle, Amer. Math. Monthly 94 (1987), 601-617. 